\title{
Увеличение порогового напряжения отпирания силовых GaN-транзисторов при использовании низкотемпературной обработки в потоке атомарного водорода
}

\author{
(C) Е.В. Ерофреев ${ }^{1}$, И.В. Федин ${ }^{2}$, И.В. Кутков ${ }^{2}$, Ю.Н. Юрьев ${ }^{3}$ \\ ${ }^{1}$ Научно-исследовательский институт систем электрической связи \\ Томского государственного университета систем управления и радиоэлектроники, \\ 634034 Томск, Россия \\ ${ }^{2}$ АО «Научно-производственная фрирма „Микран“», \\ 634045 Томск, Россия \\ ${ }^{3}$ Физико-технический институт Национального исследовательского \\ Томского политехнического университета, \\ 634050 Томск, Россия \\ E-mail: erofeev@micran.ru
}

(Получена 26 апреля 2016 г. Принята к печати 5 мая 2016 г.)

\begin{abstract}
Транзисторы с высокой подвижностью электронов на основе эпитаксиальных гетероструктур AlGaN/GaN являются перспективной элементной базой для создания устройств силовой электроники следующего поколения. Это обусловлено как высокой подвижностью носителей заряда в канале транзистора, так и высокой электрической прочностью материала, позволяющей достичь высоких напряжений пробоя. Для применения в силовых коммутационных устройствах требуются нормально-закрытые GaN-транзисторы, работающие в режиме обогащения. Для создания нормально-закрытых $\mathrm{GaN}$-транзисторов чаще всего используют подзатворную область на основе $\mathrm{GaN} p$-типа проводимости, легированного магнием $(p-\mathrm{GaN})$. Однако оптимизация толщины эпитаксиального слоя $p$ - $\mathrm{GaN}$ и уровня легирования позволяет добиться порогового напряжения отпирания $\mathrm{GaN}-$ транзисторов, близкого к $V_{\mathrm{th}}=+2 \mathrm{~B}$. В настоящей работе показано, что применение низкотемпературной обработки в потоке атомарного водорода подзатворной области на основе $p-\mathrm{GaN}$ перед осаждением слоев затворной металлизации позволяет увеличить пороговое напряжение транзистора до $V_{\text {th }}=+3.5 \mathrm{~B}$. Наблюдаемые эффекты могут быть обусловлены формированием дипольного слоя на поверхности $p$ - GaN, индуцированного воздействием атомарного водорода. Термическая обработка $\mathrm{GaN}$-транзисторов, подвергшихся водородной обработке, в среде азота при температуре $T=250^{\circ} \mathrm{C}$ в течение 12 ч не выявила деградации электрических параметров транзистора, что может быть обусловено формированием термически стабильного дипольного слоя на границе раздела металл/ $p$-GaN в результате гидрогенезации.
\end{abstract}

DOI: 10.21883/FTP.2017.02.44114.8298

\section{1. Введение}

Появившись в середине 70-х годов прошлого века, силовые MOSFETs (metal-oxide-semiconductor field effect transistors) на основе кремния $(\mathrm{Si})$ благодаря большей скорости переключения и высокой эффективности по сравнению с биполярными транзисторами стали повсеместно использоваться в силовой электронике: в источниках питания, DC-DC-преобразователях, контроллерах двигателей и пр. Однако сейчас, после непрерывного развития в течение более трех десятилетий, производительность MOSFETs на основе $\mathrm{Si}$ достигла своего теоретического предела. Следующим шагом в развитии силовой электроники стало появление мощных транзисторов на основе нитрида галлия $(\mathrm{GaN})[1]$.

Уникальные физические характеристики нитрида галлия в сравнении с кремнием позволяют достигнуть значительных усовершенствований приборов: понижения сопротивления открытого канала, роста скоростей переключения, сохранения надежности при высоких уровнях температуры и радиации, уменьшения размеров упаковки [2]. Возможность очень быстрого переключения практически без потерь, появляющаяся при использовании $\mathrm{GaN}$-транзисторов, означает возможность увеличить эффективность и уменьшить размер преобразователя, а также уменьшить себестоимость его изготовления за счет отказа от ряда дорогостоящих пассивных элементов (фильтров) схемы.

Для применения в силовой электронике необходимы нормально-закрытые GaN-транзисторы, работающие в режиме обогащения [3]. Для создания нормально-закрытых $\mathrm{GaN}$-транзисторов чаще всего используют подзатворную область на основе $\mathrm{GaN}$ р-типа проводимости, легированного магнием ( $p$-GaN).

При этом пороговое напряжение отпирания транзистора с подзатворной областью на основе $p$-GaN определяется главным образцом толщиной эпитаксиального слоя $p$ - $\mathrm{GaN}$ и уровнем его легирования. Оптимизация данных параметров позволяет добиться порогового напряжения отпирания $\mathrm{GaN}$-транзисторов, близкого к $V_{\text {th }}=+2 \mathrm{~B}$, что делает их несовместимыми с работой типовых драйверов управления кремниевыми MOSFETs. Таким образом, актуальной является задача 
повышения порогового напряжения отпирания силовых $\mathrm{GaN}$-транзисторов с подзатворной областью на основе $p$-GaN.

Известно, что выращенные с помощью метода молекулярно-лучевой эпитаксии слои $\mathrm{GaN}$, легированные магнием $(\mathrm{Mg})$, характеризуются $p$-типом проводимости [4]. В работе [5] показано, что термическая обработка слоя $p$-GaN при температуре $600^{\circ} \mathrm{C}$ в атмосфере $\mathrm{NH}_{3}$ приводит к уменьшению его слоевого сопротивления на 6 порядков, в то время как аналогичная термообработка в атмосфере $\mathrm{N}_{2}$ не приводит к заметным изменениям. Таким образом, было установлено, что взаимодействие атомов водорода с тонкими эпитаксиальными слоями $p$-GaN проводит к изменению их электрических характеристик в результате образования устойчивых соединений $\mathrm{Mg}-\mathrm{H}[6,7]$.

Целью настоящей работы является исследование влияния низкотемпературной обработки в потоке атомарного водорода подзатворной области на основе $p-\mathrm{GaN}$ перед осаждением слоев затворной металлизации на электрические характеристики нормально-закрытых силовых $\mathrm{GaN}$-транзисторов.

\section{2. Методики эксперимента}

В экспериментах использовались эпитаксиальные гетероструктуры типа $p$-GaN/AlGaN/GaN, выращенные методом металлоорганической газофазовой эпитаксии на подложках кремния диаметром 100 мм. Гетероструктура включала в себя буферный слой на основе легированного углеродом $\mathrm{GaN}$, толщиной 4 мкм, канальный слой $\mathrm{GaN}$ толщиной 400 нм, спейсер AlN толщиной 1 нм, барьерный слой $\mathrm{Al}_{0.25} \mathrm{Ga}_{0.75} \mathrm{~N}$ толщиной 20 нм и слой $p-\mathrm{GaN}$, легированный магнием. Толщина слоя $p$-GaN составляла 50 нм, концентрация атомов магния определялась методом вторично-ионной масс-спектроскопии и составляла $2 \cdot 10^{19} \mathrm{~cm}^{-3}$. Подзатворная область на основе $p$-GaN формировалась селективным плазмохимическим травлением. После формирования межприборной изоляции на пластине осуществлялось формирование омических контактов на основе $\mathrm{Ti} / \mathrm{Al} / \mathrm{Mo} / \mathrm{Au}$ и Pd-затворов.

Перед осаждением затворов на основе палладия методом электронно-лучевого испарения в вакууме образцы подвергались обработке in situ в потоке атомарного водорода с плотностью потока атомов $j>10^{16} \mathrm{~cm}^{-2} \cdot \mathrm{c}^{-1}$ в течение времени $t=10-60$ с при комнатной температуре. Для проведения экспериментов использовался источник атомарного водорода, описанный в работе [8].

Механизмы взаимодействия водорода с эпитаксиальными слоями $p$-GaN исследовались с помощью инфракрасной (ИК) спектроскопии. Электрические параметры $\mathrm{GaN}$-транзисторов по постоянному току исследовались с помощью измерителя характеристик полупроводниковых приборов НР4156А.

\section{3. Экспериментальные результаты и их обсуждение}

На рис. 1 представлено микроскопическое изображение сформированных мощных $\mathrm{GaN}$-транзисторов с шириной затворной металлизации 10 мм. Длины подзатворной области $p-\mathrm{GaN}$, а также самого затвора составляли 1.8 и 0.8 мкм соответственно. Расстояния затвор-исток и сток-исток составляли 1 и 7.5 мкм соответственно.

Транзисторы на основе эпитаксиальных гетероструктур $p-\mathrm{GaN} / \mathrm{AlGaN} / \mathrm{GaN}$ с необработанной в потоке атомарного водорода подзатворной областью $p-\mathrm{GaN}$ работали в режиме обеднения со значением порогового напряжения $V_{\text {th }}=-1.4 \mathrm{~B}$. Сопротивление канала транзистора в открытом состоянии составило $R_{\text {on }}=2.4 \mathrm{MOM} \cdot \mathrm{cm}^{2}$, а напряжение пробоя сток-исток в закрытом состоянии $V_{d-s}=250$ В при расстоянии сток-исток 7.5 мкм.

На рис. 2 представлена зависимость порогового напряжения отпирания транзистора на основе $p$-GaN/AlGaN/GaN от времени обработки в потоке атомарного водорода. Видно, что водородная обработка подзатворной области $p$-GaN в течение $t=10$ с при

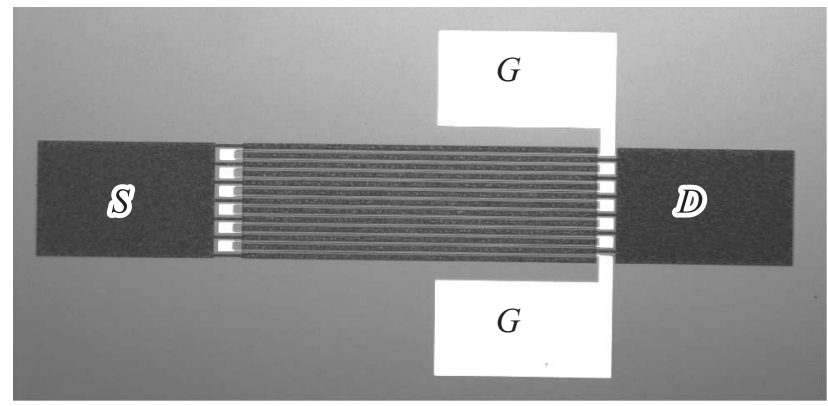

Рис. 1. Микроскопическое изображение транзистора на основе $p$-GaN/AlGaN/GaN/Si с шириной затвора 10 мм. $S$ - исток, $D$ - сток, $G$ - затвор.

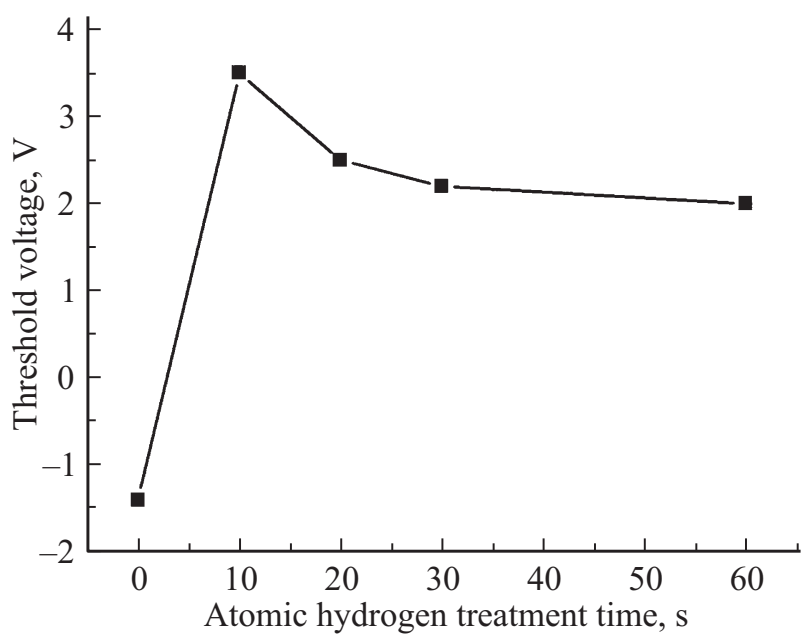

Рис. 2. Зависимость порогового напряжения транзистора на основе $p$-GaN/AlGaN/GaN от времени обработки в потоке атомарного водорода. 


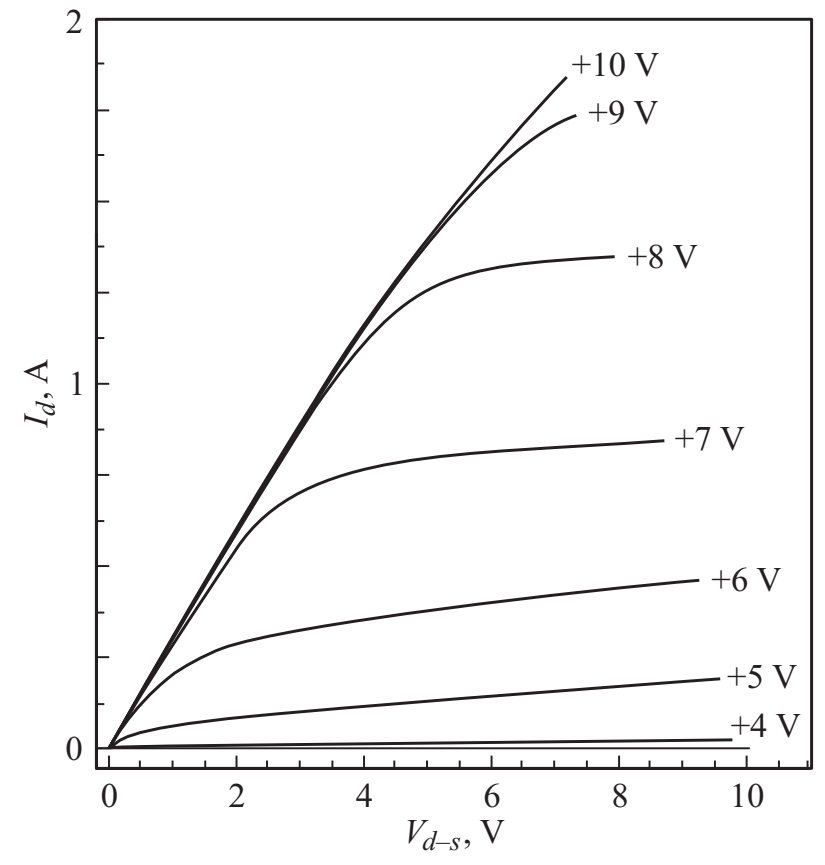

Рис. 3. Выходная характеристика транзистора на основе $p-\mathrm{GaN} / \mathrm{AlGaN} / \mathrm{GaN} / \mathrm{Si} \mathrm{c} \mathrm{шириной} \mathrm{затвора} 10 \mathrm{Mм}$, изготовленного с применением водородной обработки в течение $t=10 \mathrm{c}$.

комнатной температуре перед формированием затворной металлизации на основе пленки палладия позволяет увеличить пороговое напряжение отпирания $\mathrm{GaN}$-транзистора до $V_{\text {th }}=+3.5$ В. При этом дальнейшее увеличение времени обработки с $t=10$ до 60 с приводит к уменьшению данной величины до $V_{\text {th }}=+2 \mathrm{~B}$, что может быть обусловлено проникновением (диффузией) атомов водорода в глубь подзатворной области на основе $p$-GaN толщиной 50 нм.

На рис. 3 представлены вольт-амперные характеристики $I_{d}\left(V_{d-s}\right) \mathrm{GaN}$-транзистора, полученного при использовании кратковременной $(t=10 \mathrm{c})$ обработки подзатворной области $p$-GaN в потоке атомарного водорода. Транзистор имеет электрические характеристики, аналогичные характеристикам GaN-транзисторов, изготовленных без применения водородной обработки, однако работает в режиме обогащения с величиной порогового напряжения отпирания $V_{\mathrm{th}}=+3.5 \mathrm{~B}$ и током насыщения сток-исток $I_{\mathrm{sat}}=0.2 \mathrm{~A} / \mathrm{Mм}$ при напряжении затвор-исток $V_{g-s}=+10 \mathrm{~B}$.

На рис. 4 и 5 представлены результаты исследования методом ИК спектроскопии поглощения поверхности слоев $p-\mathrm{GaN}$ толщиной $50 \mathrm{Hм}$, без обработки и прошедших обработку слоя $p$-GaN в потоке атомарного водорода в течение $t=10$ и $60 \mathrm{c}$ при комнатной температуре. Данные рис. 4 и 5 свидетельствуют о том, что обработка подзатворной области $p$-GaN в потоке атомарного водорода при комнатной температуре в течение $t=10$ с приводит к формированию соединений $\mathrm{Mg}-\mathrm{H}$ на поверхности, а также уменьшению концентрации акцепторов $\mathrm{Mg}$ (уровня легирования) в объеме $p-\mathrm{GaN}$. Увеличение времени водородной обработки до $t=60 \mathrm{c}$ приводит к стимулированному росту количества связей $\mathrm{Mg}-\mathrm{H}$ и дальнейшему уменьшению уровня легирования слоя $p$-GaN.

Для объяснения наблюдаемых эффектов может быть предложен следующий механизм. После кратковременной $(t=10 \mathrm{c})$ обработки подзатворной области на основе $p$-GaN в потоке атомарного водорода на поверхности полупроводника в результате образования связей $\mathrm{Mg}-\mathrm{H}$ формируется дипольный слой, индуцированный водородом. Наличие данного дипольного слоя на границе раздела $\mathrm{Pd} / p-\mathrm{GaN}$ приводит к росту порогового напряжения отпирания $\mathrm{GaN}$-транзистора посредством увеличения высоты барьера Шоттки [9].

Дальнейшее увеличение времени обработки в потоке атомарного водорода приводит к его проникновению в слой $p$-GaN, а также увеличению количества сформированных связей $\mathrm{Mg}-\mathrm{H}$ на поверхности. Диффузия атомов

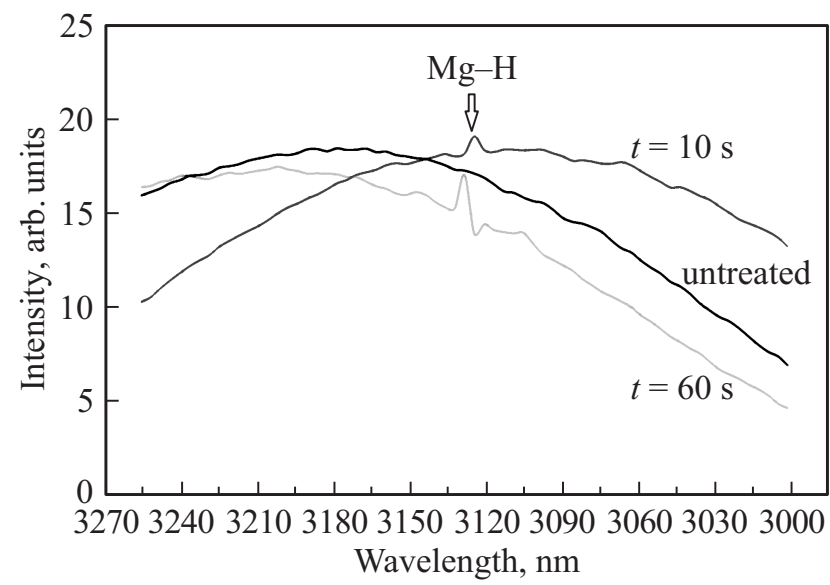

Рис. 4. Поглощение связями $\mathrm{Mg}-\mathrm{H}$ в ИК спектрах необработанных слоев $p$-GaN и слоев $p$-GaN, подвергшихся обработке в потоке атомарного водорода в течение 10 и $60 \mathrm{c}$.

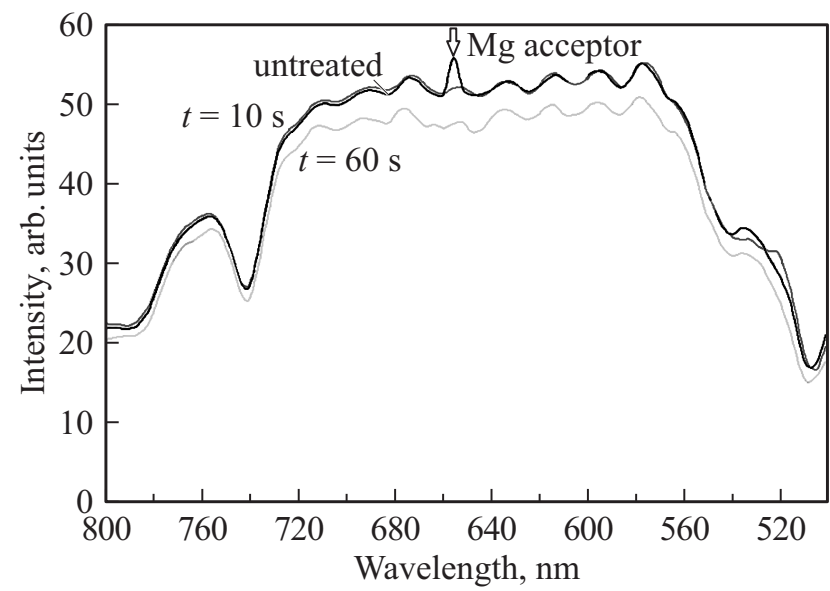

Рис. 5. Поглощение акцепторами $\mathrm{Mg}$ в ИК спектрах необработанных слоев $p$-GaN и слоев $p$-GaN, подвергшихся обработке в потоке атомарного водорода в течение 10 и $60 \mathrm{c}$. 


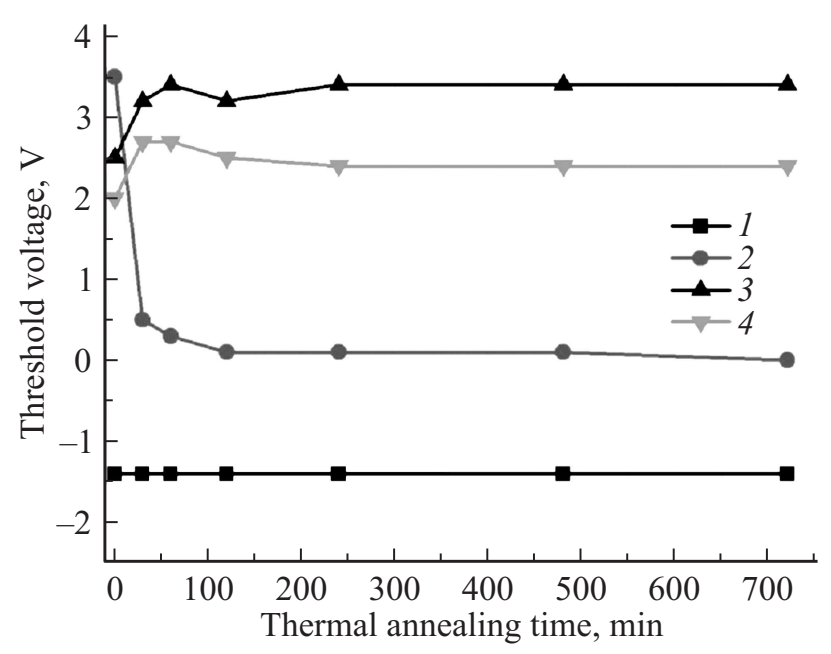

Рис. 6. Зависимость от времени термической обработки в среде азота при $250^{\circ} \mathrm{C}$ порогового напряжения транзисторов без обработки в атомарном водороде (1) и подвергшихся обработке в потоке атомарного водорода в течение $t=10(2)$, $20(3), 60 \mathrm{c}(4)$.

водорода в глубь слоя $p-\mathrm{GaN}$ приводит к снижению уровня его легирования, что в свою очередь приводит к последующему уменьшению порогового напряжения отпирания транзистора.

На рис. 6 представлены результаты исследования термической стабильности порогового напряжения отпирания $\mathrm{GaN}$-транзисторов без обработки (кривая 1) и прошедших обработку в потоке атомарного водорода слоя $p-\mathrm{GaN}$ в течение $t=10,20$ и 60 с (кривые 2-4) при комнатной температуре. Термическая обработка проводилась при температуре $250^{\circ} \mathrm{C}$ в течение $t=0.5-12$ ч в среде азота.

Данные рис. 6 свидетельствуют о том, что термическая обработка $\mathrm{GaN}$-транзисторов с подзатворной областью $p$-GaN, обработанной в течение $t=10$ с в потоке атомарного водорода, приводит к снижению величины порогового напряжения с $V_{\text {th }}=+3.5 \mathrm{~B}$ до $+0.5 \mathrm{~B}$. При этом в $\mathrm{GaN}$-транзисторах с подвергшимся водородной обработке слоем $p$-GaN в течение $t=20$ и 60 с заметной деградации порогового напряжения не наблюдается.

Высокая термическая стабильность, наблюдаемая на рис. 6, может быть объяснена формированием индуцированного водородом термостабильного дипольного слоя на границе раздела металл/p-GaN.

\section{4. Заключение}

Мощные GaN-транзисторы на основе эпитаксиальных гетероструктур $\mathrm{AlGaN} / \mathrm{GaN}$, работающие в режиме обогащения, являются перспективной элементной базой для создания устройств силовой электроники следующего поколения. Для получения нормально-закрытных
$\mathrm{GaN}$-транзисторов чаще всего используется подзатворная область на основе слоя $p$-GaN. При этом оптимизация его толщины и уровня легирования позволяет достичь величины порогового напряжении отпирания транзистора $V_{\text {th }}=+2 \mathrm{~B}$.

В настоящей работе показано, что использование кратковременной низкотемпературной обработки в потоке атомарного водорода подзатворной области $p$-GaN перед осаждением слоев затворной металлизации позволяет увеличить пороговое напряжение отпирания транзистора до $V_{\text {th }}=+3.5 \mathrm{~B}$. Наблюдаемые эффекты могут быть обусловлены формированием дипольного слоя на поверхности $p$ - $\mathrm{GaN}$, индуцированного воздействием атомарного водорода.

Термическая обработка $\mathrm{GaN}$-транзисторов, подвергшихся водородной обработке в среде азота при температуре $250^{\circ} \mathrm{C}$ в течение $t=12$ ч, не выявила деградации электрических параметров транзистора, что может быть обусловлено формированием термически стабильного дипольного слоя на границе раздела металл/p-GaN в результате гидрогенизации.

Авторы работы выражают благодарность коллективу Научно-производственного комплекса „Микроэлектроника“ АО Научно-производственной фирмы „Микран“ и коллективу Научно-образовательного центра „Нанотехнологии“ Томского государственного университета систем управления и радиоэлектроники за содействие в проведении экспериментальной части работы и обсуждение результатов.

Работа выполнена при финансовой поддержке Министерства образования и науки РФ в рамках соглашения № 14.577.21.0204 от 27.10.15 II: уникальный идентификатор проекта RFNEFI57715X0204.

\section{Список литературы}

[1] M. Briere. GaN-based Power Device Platform. The arrival of a new paradigm in conversion technology. www.powersystemdesign.com

[2] M. Germain, K. Hodson, H. Kawai, T. Kobayashi, E. Ysewijn. Power Devices, 4, 6 (2012).

[3] J. Würfl, O. Hilt, E. Bahat-Treidel, R. Zhytnytska, K. Klein, P. Kotara, F. Brunner, A. Knauer, O. Krüger, M. Weyers, G. Tränkle. ECS Trans., 52 (1), 979 (2013).

[4] T.D. Moustakas, R. Molnar. Mater. Res. Soc. Symp. Proc., 281, 753 (1993).

[5] S. Nakamura, N. Ivasa, M. Senoh, T. Mikai. Jpn. J. Appl. Phys., 31, 1258 (1992).

[6] J. Neugebauer, C.G. Van de Walle. Phys. Rev. Lett., 75, 4452 (1995).

[7] W. Gotz, N.M. Johnson, J. Walker, D.P. Bour, R.A. Street. Appl. Phys. Lett., 68, 667 (1996).

[8] Е.В. Ерофеев, В.А. Кагадей. Микроэлектроника, 41 (2), 1 (2012).

[9] G. K. Reeves, H. B. Harrison. IEEE Electron Dev. Lett., 3 (5), 111 (1982).

Редактор Л.В. Шаронова 


\title{
Improve of the threshold voltage of high voltage GaN transistors by low temperature atomic hydrogen treatment
}

\author{
E.V. Erofeev' ${ }^{1}$, I.V. Fedin ${ }^{2}$, I. Kutkov' ${ }^{2}$, Y. Yuryev ${ }^{3}$ \\ ${ }^{1}$ Research Institute \\ of Electrical Communication Systems \\ of Tomsk State University \\ of Control Systems and Radioelectronics, \\ 634050 Tomsk, Russia \\ 2 Research \& Production Company „Micran“, \\ 634041 Tomsk, Russia, \\ 3 Institute of Physics and Technology, \\ National Research Tomsk Polytechnic University, \\ 634050 Tomsk, Russia
}

Abstract $\mathrm{AlGaN} / \mathrm{GaN}$ HEMT is one of attractive candidates for next generation high power devices because of high carrier mobility in two-dimentional electron gas channels and high breakdown voltage. In order to apply the AlGaN/GaN HEMTs for power switching applications the normally off operation is required. Enhancement type behavior of GaN HEMT transistors is obtained by using $p$-type $\mathrm{Mg}$-doped $\mathrm{GaN}$ gate structures. The optimized epitaxial designs enable threshold voltage close to $V_{\text {th }}=+2 \mathrm{~V}$. In present work, it is shown that atomic hydrogen treatment of the $\mathrm{Mg}$ doped $p-\mathrm{GaN}$ before gate metal evaporation can increase the threshold voltage up to $V_{\text {th }}=+3.5 \mathrm{~V}$. It can be caused by the hydrogen-induced dipole layer formation at the $p$-GaN semiconductor interface after atomic hydrogen treatment. No visible parameters degradation after thermal annealing at $T=250^{\circ} \mathrm{C}$ for $12 \mathrm{~h}$ in nitrogen environment was observed. It can be explained by the formation thermally stable $\mathrm{Mg}-\mathrm{H}$ complexes in the $p$-GaN layer after hydrogenation. 